\title{
The Pro-inflammatory Markers and Cytokine Profile in Acute Chikungunya Virus Infections in a Rural Community from North India
}

\author{
D Chattopadhya ${ }^{1}$, A Verghese ${ }^{2}$, S Broor ${ }^{3}$
}

\begin{abstract}
Analysis of pro-inflammatory markers, i.e., nitrite, citrulline, tumor necrosis alfa (TNF- $\alpha$ ) and Th1 and Th2-specific cytokines, viz., IL-2 and IL-4 respectively were analyzed in 30 sera positive for IgM antibodies to Chikungunya virus (CHIKV) from a rural hospital-attending population in comparison to 40 sera from cases with other febrile illness (OFI) and 30 healthy controls.

Levels of nitrite, citrulline, TNF- $\alpha$ and IL-4 were found to be increased in serum while serum level of IL-2 was found to be depressed in anti-CHIKV IgM positive cases compared to OFI and control groups.

The serum nitrite levels in anti-CHIKV IgM positive cases showed positive correlation with citrulline, TNF- $\alpha$ and IL-2 and negative correlation with IL-4 level. The IL-2 level showed negative correlations with that of IL-4 level in serum.
\end{abstract}

Keywords: Chikungunya virus, Nitrite, TNF- $\alpha$, Cytokines

\section{Introduction}

Chikungunya virus (CHIKV) is an arthropod-borne RNA virus that belongs to Alphavirus genus in the Togaviridae family. ${ }^{1}$ Recently, outbreaks of this infectious disease have emerged in various countries of South-East Asia including India. ${ }^{2,3}$ Most reports on CHIKV infection so far have been focused on the epidemiology and virus genotyping. There are a few reports on immune alteration induced by this virus, especially during the acute phase that may have prognostic implications on the future disease course. ${ }^{4}$

Macrophages are readily infected by CHIKV at a very early stage. ${ }^{5,6}$ Production of nitric oxide (NO) by macrophages reflects a pro-inflammatory response that is instrumental in mounting innate immunity against viral infections. ${ }^{7}$ Measurement of nitrite level in serum is commonly employed as an indicator of NO production due to short life span of the later ( $5-6 \mathrm{sec}){ }^{7}$ Estimation of citrulline is commonly employed as a surrogate marker for validating specificity of nitric oxide production through arginine-dependent pathway due to identical pathway of production for both nitric oxide and citrulline. ${ }^{8}$ Tumor necrosis alpha (TNF- $\alpha$ ) is a pro-inflammatory cytokine that is released from macrophages in response to viral infections. ${ }^{9}$ On the other hand, cytokines released by T helper and T suppressor lymphocytes are strong

${ }^{1}$ Assistant Professor, ${ }^{3}$ Professor and Head, Department of Microbiology, Faculty of Medical and Health Sciences, SGT University, Gurgaon - 122505, India.

${ }^{2}$ Assistant Research Officer, Department of Microbiology, National Center for Disease Control, 22,Sham NathMarg, Delhi - 110054,India.

Correspondence: Dr. D Chattopadhya, Department of Microbiology, Faculty of Medical and Health Sciences, SGT University, Gurgaon - 122505, India.

E-mail Id: dchattopadhya27@gmail.com

Orcid Id: http://Orcid.org/0000-0002-4117-3050

How to cite this article: Chattopadhya D, Verghese A, Broor S. The Pro-inflammatory Markers and Cytokine Profile in Acute Chikungunya Virus Infections in a Rural Community from North India. J Commun Dis 2017; 49 (3): 67-72.

Digital Object Identifier (DOI): 10.24321/0019.5138.201725

ISSN: 0019-5138 
indicators of adaptive immunity. ${ }^{10}$ Imbalance in the profile between cytokines released by T-helper type-1 (Th1) lymphocytes and that released by T helper type-2 (Th2) lymphocytes has been linked with pathogenesis of dengue virus (DENV) infection, which is a closely related arboviral infection. ${ }^{11,12}$ However, CHIKV is characterized by much less severe clinical manifestation and mortality compared to DENV infection except musculoskeletal involvement, suggesting a difference in the role played by the immune system in these two infections. A study was taken up to analyze serum levels of nitrite, citrulline, TNF- $\alpha$ and two cytokines, viz., IL-2 and IL-4 as representatives of Th1 and Th2 categories of cytokines in CHIKV infection during the acute phase.

\section{Materials and Methods}

The project was conducted by the Department of Microbiology of SGT hospital, a tertiary care center located in a rural area of district Gurgaon, catering predominantly to the local rural community. Prior approval was obtained from institutional ethics committee constituted by SGT university comprising of external experts as members (communication dated 13.01.16). Patient consent form was not employed separately since the study utilized serum samples submitted to the laboratory for investigation by various clinical departments and which were coded, maintaining anonymity.

During the months of August and September in the year 2016, serum samples from suspected cases of CHIKV infection attending various clinical departments of SGT Hospital, Gurgaon, were received in the microbiology laboratory for serodiagnosis of CHIKV infection. The samples were screened for anti-CHIKV IgM antibody by lateral flow chromatographic immunoassay (CTK Biotech Inc, San Diego, CA, USA). The sera positive for anti-CHIKV IgM antibody were also screened for malaria (by peripheral blood smear and immune-chromatography) and DENV infection (by serology) to exclude these co-infections.

All sera positive for anti-CHIKV IgM antibodies were subjected to estimation of pro-inflammatory markers, i.e., C-reactive protein, ferritin, nitrite, citrulline and TNF- $\alpha$ as well as estimation of Th1 and Th2-specific cytokines, i.e., IL-2 and IL-4 respectively.

A total of 40 sera from age and sex-matched febrile cases screened negative for DENV and CHIKV infections by serology and negative for malaria infection by peripheral smear as well as by immune-chromatography were included in the study as a group defined as 'Other Febrile Illness (OFI)'. A total of 30 age and sex-matched healthy blood donors from local community were included as controls.

\section{Estimation of C-reactive protein and ferritin}

Detection of C-reactive protein in sera was carried out using commercial kit (Angstrom Biotech, Vadodora, India) based on the principle of latex agglutination test. Semiquantitative estimation of CRP was expressed in terms of the highest dilution in doubling dilution of serum (expressed as $\log _{2}$ titer) giving positive result. Level of ferritin was measured by enzyme-linked immunosorbent assay (ELISA) kit (Calbiotech, CA, USA).

\section{Estimation of Serum Levels of Nitrite and Citrulline}

Estimation of serum levels of nitrite was based on Griess reaction. ${ }^{13}$ Briefly, serum sample was diluted four-fold in distilled water and de-proteinized by adding zinc sulfate (final concentration $15 \mathrm{~g} / \mathrm{L}$ ) followed by centrifugation at $10,000 \mathrm{~g}$ for $5 \mathrm{~min}$ at room temperature. One hundred microliter of supernatant as well as $100 \mu \mathrm{L}$ of varying dilutions of nitrite standards (Sigma Chemicals, USA) was charged in a flat-bottom 96-well plate. One hundred microliter of Greiss reagent $(0.1 \%$ napthylene-diaminedihydrochloride, $1 \%$ sulphanilamide and $2.5 \%$ phosphoric acid) was added to each well. The plate was incubated for $10 \mathrm{~min}$ at room temperature in dark and was read at $550 \mathrm{~nm}$ in an automated plate reader (Multiskan, Lab systems). Quantity of nitrite in samples was calculated from absorbance values plotted against the standard curve obtained from the values of the serial dilutions of nitrite standards using reading of the reaction mixture without serum as blank. Each sample was run in triplicate and average value taken. The lower limit of detection of nitrite concentration in serum was $250 \mathrm{nmol} / \mathrm{L}$.

Estimation of serum level of citrulline was carried out by colorimetric estimation based on the protocol described by Boyde and Rahmatulla on the basis of the chemical modification of citrulline by deacetyl-monoxime. ${ }^{14}$ Briefly, serum sample was de-proteinized by adding tricarboxylic acid solution (final concentration $5 \%$ ) followed by centrifugation. Chromogenic reagent was prepared just before use by adding $5 \mathrm{mg}$ thiosemicarbazide (Sigma Chemicals, USA) to $50 \mathrm{~mL}$ of $0.5 \%$ diacetyl-monoxime (Sigma Chemicals, USA) solution followed by addition of $100 \mathrm{~mL}$ of acid-ferric solution prepared by dissolving $\mathrm{FeCl}_{3}(25 \mathrm{mg}$ ) in $100 \mathrm{~mL}$ solution containing $25 \mathrm{~mL}$ concentrated sulfuric acid (95-98\%), $20 \mathrm{~mL}$ concentrated phosphoric acid (85\%) and $55 \mathrm{~mL}$ distilled water. To 100 $\mu \mathrm{L}$ of supernatant, $3 \mathrm{~mL}$ of chromogenic solution was added, mixed vigorously and boiled at $100^{\circ} \mathrm{C}$ for $5 \mathrm{~min}$. Enzyme Urease (Type VII, Sigma Chemicals, USA) was included in the mixture to prevent formation of color complex by urea with diacetyl-monoxime. The tubes were cooled to room temperature and absorbance was measured at $530 \mathrm{~nm}$ in a spectrophotometer. Quantity of citrulline in supernatant was calculated from a standard curve of absorbance values prepared by running varying dilutions of DL-citrulline standard (Sigma Chemicals, USA) simultaneously with samples. Representative samples (40\%) were also analyzed by high-performance liquid 
chromatography (HPLC) (Shimadju Corporation, Japan) to validate sensitivity of the colorimetric assay ( $<5 \%$ variation). The detection limit of citrulline was $2.5 \mu \mathrm{mol} / \mathrm{L}$.

Serum levels of TNF- $\alpha$, IL-2 and IL-4 were measured using commercial kits (R\&D Systems, USA) with detection limits of $0.38 \mathrm{pg} / \mathrm{mL}, 3 \mathrm{pg} / \mathrm{mL}$ and $0.22 \mathrm{pg} / \mathrm{mL}$ respectively. The coefficient of variation was detected to be less than $10 \%$ for all the kits. Specificity of the kits was checked using heterogeneous recombinant cytokines. Inter-assay and intra-assay variation for all of the assays was found to be less than $10 \%$. Serum sample showing undetectable level of cytokine was ascribed $0 \mathrm{pg} / \mathrm{mL}$ value for the purpose of the present study. ${ }^{15}$

\section{Statistical Analysis}

The differences in serum cytokines for the overall group was done calculated by ANOVA while the same between the groups were compared by a non-parametric analysis with Kruskal-Wallis test with level of significance at $p<0.05$. Correlations between continuous variables were measured by calculating Pearson's correlation coefficient with linear regression analysis.

\section{Results}

A total of 30 out of 200 consecutive sera were detected to be positive for anti-CHIKV IgM antibody during the study duration. The age group of the positive cases ranged between 5 and 65 years with male: female ratio as 1.3:1.

A total of 9 out of 30 sera (30\%) in the anti-CHIKV IgMpositive group were detected to be positive for CRP, with mean $\left(\log _{2}\right)$ titer of 5.66 while only one out of 40 sera (2.5\%) in the OFI group and none of the sera in the control group showed positivity for CRP. Level of serum ferritin ( $\mathrm{mg} / \mathrm{L}$ ) was significantly increased in the anti-CHIKV IgM-positive cases (mean \pm SD as $101 \pm 60.8$ ) compared to OFI group (mean \pm SD as $68 \pm 20.4$ ) as well as control group (mean \pm SD as $46 \pm 11.3$ ), $p<0.001$ and $<0.001$ respectively (data not shown in Table 1). There was a positive correlation $(r=0.679 ; p<0.01)$ between the titer of CRP and serum level of ferritin in the cases positive for CRP.

Levels of nitrite, citrulline, TNF- $\alpha$ and IL- 4 were found to be increased in serum while serum level of IL-2 was found to be depressed in anti-CHIKV IgM-positive cases compared to OFI and control group (Table 1).

Table 1.Serum Levels of Pro-inflammatory Markers and Cytokines in Acute CHIKV Infection

\begin{tabular}{|c|c|c|c|c|c|c|c|}
\hline \multirow[t]{2}{*}{$\begin{array}{c}\text { Serum Levels } \\
\text { (Mean } \pm S D)\end{array}$} & \multirow{2}{*}{$\begin{array}{c}\text { Anti-CHIKV IgM Antibody } \\
\text { Positive Group }(n=30) \\
\text { Group I }\end{array}$} & \multirow{2}{*}{$\begin{array}{l}\text { OFI Group } \\
\text { (n=40) } \\
\text { Group II }\end{array}$} & \multirow{2}{*}{$\begin{array}{l}\text { Controls } \\
(n=30) \\
\text { Group III }\end{array}$} & \multicolumn{4}{|c|}{$\begin{array}{l}\text { Statistical Comparisons between } \\
\text { Groups }\end{array}$} \\
\hline & & & & Overall & | Vs || & I Vs III & II Vs III \\
\hline Nitrite $(\mu \mathrm{mol} / \mathrm{L})$ & $10.6 \pm 6.4$ & $5.1 \pm 2.1$ & $4.4 \pm 2.6$ & $<0.001$ & $<0.001$ & $<0.001$ & 0.257 \\
\hline Citrulline $(\mu \mathrm{mol} / \mathrm{L})$ & $34.7 \pm 6.6$ & $18 \pm 8.2$ & $20.4 \pm 8.8$ & $<0.001$ & $<0.001$ & $<0.001$ & 0.277 \\
\hline TNF- $\alpha(p g / m L)$ & $18.8 \pm 14.6$ & $3.3 \pm 1.7$ & $1.5 \pm 1.1$ & $<0.001$ & $<0.001$ & $<0.001$ & $<0.001$ \\
\hline $\mathrm{IL}-2$ (pg/mL) & $6.1 \pm 5.0$ & $12.1 \pm 5.4$ & $16.6 \pm 3.1$ & $<0.001$ & $<0.001$ & $<0.001$ & $<0.001$ \\
\hline $\mathrm{IL}-4(\mathrm{pg} / \mathrm{mL})$ & $13.9 \pm 12.2$ & $3.2 \pm 1.3$ & $1.4 \pm 0.8$ & $<0.001$ & $<0.001$ & $<0.001$ & $<0.001$ \\
\hline
\end{tabular}

The anti-CHIKV IgM-positive group showed positive correlation of serum nitrite levels with levels of citrulline ( $r=0.801 ; p<0.001$, not shown in Fig. 1). Serum nitrite level also showed positive correlation with TNF- $\alpha$ (Fig. 1) and
IL-2 (Fig. 2) but negative correlation with serum IL-4 (Fig. 2). A negative correlation was observed between serum levels of IL-2 and IL-4 (Fig. 2).

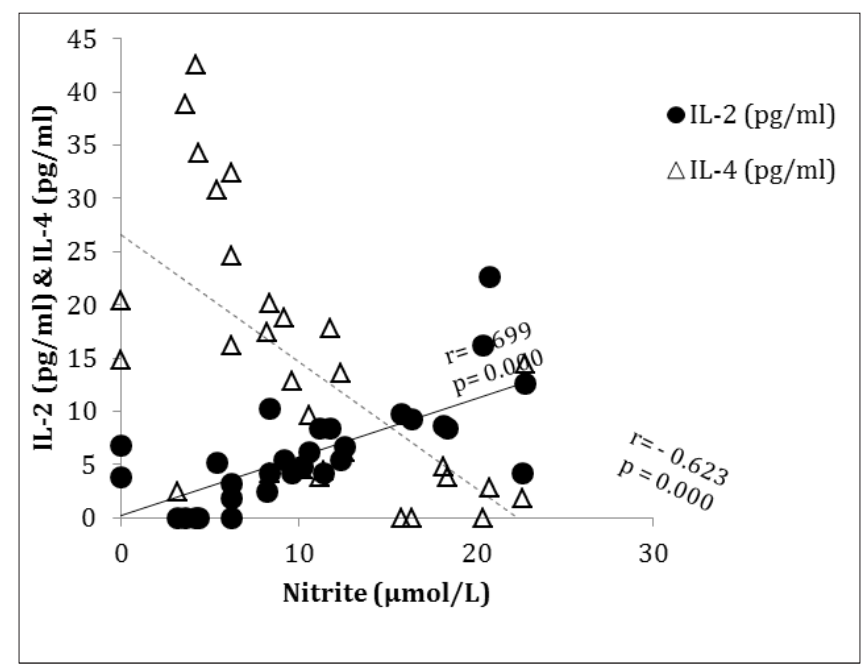

Figure 1.Correlation between Nitrite and TNF- $\alpha$ Levels in Serum in Acute CHIKV Infection 


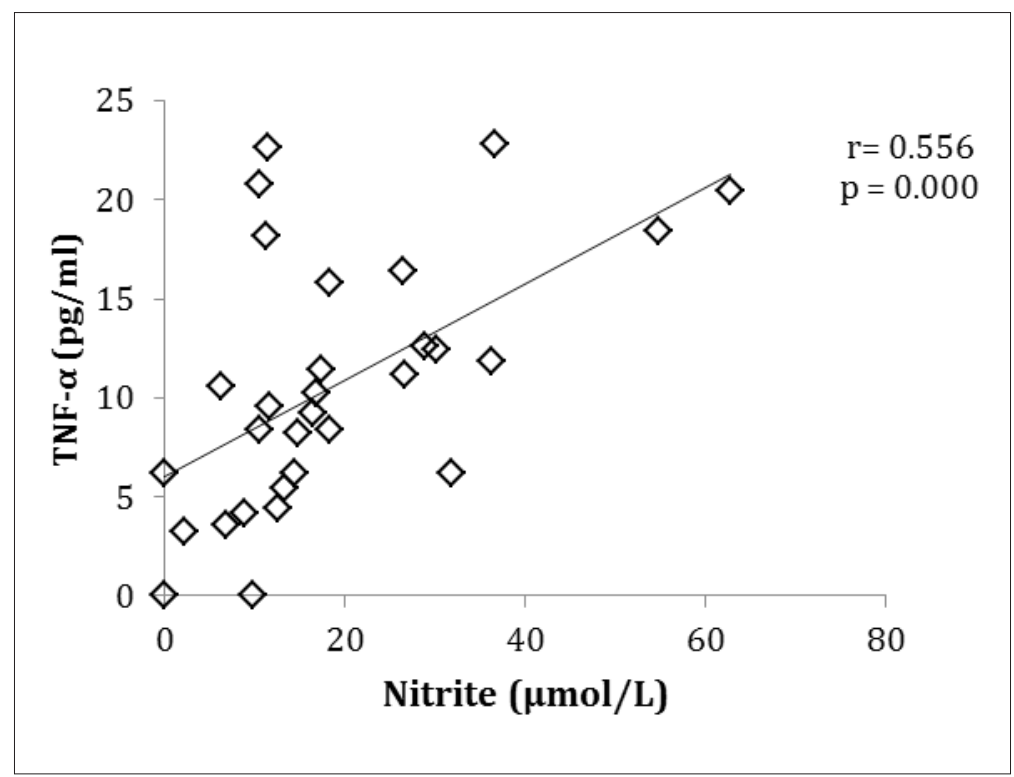

Figure 2.Correlations between Nitrite and IL-2 as well as IL-4 Levels in Serum in Acute CHIKV Infection

\section{Discussion}

$\mathrm{CHIKV}$ infection is no longer considered an urban problem and spread of CHIKV infection to the rural community has been reported from India. ${ }^{16,17}$ The vector of CHIKV, i.e., Aedes albopictus, exploits wider range of larval habitats, both natural and artificial, compared to Aedes aegyptii explaining its abundance in rural areas. ${ }^{18}$ Rapid urbanization, storage of water in cemented containers, sleeping habit with body parts uncovered, ignorance about preventive measures have all been considered to be contributory to the problem of CHIKV in the rural community. ${ }^{16,17}$ In the present study, anti-CHIKV IgM was detected in $15 \%$ by immune-chromatography in acute phase sera from rural community with clinical suspicion of CHIKV infection. There is limited information on incidence of CHIKV in a rural community. Studies from rural western India reported IgM positivity as $17 \%$ and $32.8 \%$ by immune-chromatography and capture ELISA respectively. ${ }^{17,19}$ The lower positivity rate in the present report could be due to the difference in the period of study that might have been beyond the peak epidemic season in the population. To the best of our knowledge, there is no report from this part of the country, i.e., Northern India on seroprevalence of CHIKV infection in rural community.

Higher frequency of CRP as well as higher levels of ferritin detected in anti-CHIKV IgM-positive cases in the present study is in accordance with reports that acute phase reactants are elevated in dengue fever as well as in CHIKV infections, ${ }^{20}$ although admittedly in absence of follow-up data available with us, it was not possible to correlate such alterations with joint manifestations. The level of nitrite as a pro-inflammatory marker was found to be elevated in sera in our study with anti-CHIK IgM positivity suggesting activation of macrophages in the acute stage of CHIKV infection as an attempt to mount innate immune response, ${ }^{4}$ supporting the common description of the CHIKV infection as an inflammatory disease. ${ }^{21}$ Concomitant elevation of citrulline level proportionate with elevated nitrite level confirmed involvement of arginine pathway towards production of NO. Production of NO by the macrophages that plays an important role in antiviral defense is regulated by inducible nitric oxide synthase (iNOS). ${ }^{22} \mathrm{TNF}-\alpha$, another proinflammatory cytokine released by activated macrophages, is also considered to play an important role in defense against viral infections. ${ }^{23}$ In fact, therapeutic blockade of TNF- $\alpha$, trialed for rheumatoid arthritis, has been reported to result in increased susceptibility to a number of viral infections, viz., human immunodeficiency virus, varicella zoster, papilloma and cytomegalovirus. ${ }^{24}$ Thus, positive correlations between elevated levels of nitrite, citulline and TNF- $\alpha$ levels reflect a concerted pro-inflammatory response by macrophages to mount innate defense against CHIKV infection.

However, the anti-CHIKV IgM-positive group in the present study also demonstrated elevation of Th2-specific cytokine, viz., IL-4 and a depression of Th1-specific cytokine, viz., IL2. Limited studies reported so far on analysis of cytokine profile during acute phase of CHIKV infection have yielded contradictory result that has mostly been attributed to the difference in disease stages of patients included in each study. ${ }^{25}$ The present study employed IL-2 and IL-4 as markers for evaluating Th1 and Th2 types of immune responses respectively with the consideration that many other members of Th1 and Th2 categories of the cytokines have relatively wider spectrum of sources other than Th1 or Th2 lymphocytes. For instance, IFN- $\gamma$ and IL-10 that have been employed as markers for studying Th1 and Th2 types of response respectively are also known to be produced by macrophages apart from T lymphocytes. ${ }^{26,27}$ The Th1 cytokine IL-2 is known to promote killing of virus-infected cells, whereas Th2 cytokine IL-4 induces antibody response 
involved in viral neutralization. ${ }^{11,12}$ Thus, elaboration of Th2 category of cytokines, as observed in the present study, may be a reflection of adaptive immunity towards production of specific antibody by B cells. In comprehensive studies by Thangamani et al. ${ }^{27}$ and $\mathrm{Ng}$ et al. ${ }^{28}$ acute $\mathrm{CHIKV}$ infection demonstrated dominance of Th2-mediated response with elevated expression levels of IL-4 and IL-10 and depressed Th1-mediated response as evident by decreased levels of IFN- $\gamma$ and IL-2. Elevation of Th2-specific cytokines, as observed in the present study during acute phase with IgM positivity, may have prognostic significance as IgM to antiCHKV associated with Th2 dominant cytokine profile has been associated with delay in resolution of musculoskeletal symptoms. ${ }^{20}$

Considering the fact that the Th2 category cytokines are anti-inflammatory in nature, our observation on simultaneous elevation in levels of nitrite that is considered indicator of pro-inflammatory response apparently depicts a contradictory finding. Reports on the time kinetics of antibody response have been reported to be variable in CHIKV infection with some describing such response to be concomitant along with innate immunity while others have observed appearance of adaptive response following switch from innate immunity. ${ }^{17,19,30}$ It has been hypothesized that an inflammatory response might occur earlier when the virus is actively replicating and then gets down-regulated by a counter anti-inflammatory response when the virus is being eliminated from circulation. ${ }^{21,23,30}$ Closer analysis of the values of IL-2 and IL-4 individually revealed wide scatter in levels of both IL-2 and IL-4 with many cases having values either lower or higher compared to controls for both the parameters. Thus, considering the definite negative correlation observed between IL-4 level and both nitrite and IL-2 levels, it may be possible that the cases in our study were in transition phase from a pro-inflammatory status to anti-inflammatory one with some in still pro-inflammatory phase and some in the later phase. Admittedly, a follow up of the former group of cases would have yielded more convincing evidence in favor of such postulation that has been a limitation of our study. Nevertheless, the present study provides some insight into the immune response of acute CHIKV infection in a rural community.

\section{Conclusion}

Despite significant elevation of pro-inflammatory response in terms of elevated levels of nitrite and TNF- $\alpha$, acute CHIKV infection may show dominance of Th2 (anti-inflammatory) type of cytokine profile over Th1 (pro-inflammatory) type. Although IgM positivity associated with Th2-dominant cytokine profile has been considered to be predictive of delay in resolution of musculoskeletal symptoms, the dynamic shift from Th1 to Th2 response in course of infection, single-point estimation of cytokine profile should not be correlated with disease outcome.

\section{Funding}

This research received no specific grant from any funding agency in the public, commercial or non-profit sectors.

\section{Conflict of Interest: None}

\section{References}

1. Ravi V. Re-emergence of Chikungunya virus in India. Indian J Med Microbiol 2006; 24: 83-84.

2. Lahariya C, Pradhan SK. Emergence of Chikungunya virus in Indian subcontinent after 32 years: A review. J Vector Borne Dis 2006; 43: 151-60.

3. Cecilia D. Current status of dengue and Chikungunya in Delhi. WHO South East Asia J Public Health 2014; 3: 22-27.

4. Wauquier N, Becquart P, Nkoghe $D$ et al. The acute phase of Chikungunya virus infection in humans is associated with strong innate immunity and T CD8 cell activation. J Infect Dis 2011; 204: 115-23.

5. Couderc T, Chrétien F, Schilte $C$ et al. Mouse model for Chikungunya: Young age and inefficient type-1 interferon signaling are risk factors for severe disease. PLoS Patho 2008; 4: e 29.

6. Ziegler SA, Lu L, Travassos RA et al. An animal model for studying pathogenesis of Chikungunya virus infection. Am J Trop Med 2008; 79: 133-39.

7. Akaike T, Maeda H. Nitric oxide and viral infection. Immunology 2000; 101: 300-08.

8. Burgner D, Rockett K, Kwiatkowski D. Nitric oxide and infectious diseases. Arch Dis Child 1999; 81: 185-88.

9. Newton K, Dixit VM. Signaling in Innate Immunity and Inflammation. Cantley L, Hunter T, Sever R et al., (edit) in Cold Spring Harb Perspect Biology 2012; 4: a006049.

10. Chaplin DD. Overview of immune response. J Allergy Clin Immunol 2010; 125(Suppl 2): S3-S23.

11. Chaturvedi UC, Raghupathy R, Pacsa AS et al. Shift from a Th1-type response to Th2-type in dengue hemorrhagic fever. Curr Sci 1999; 76: 63-69.

12. Chaturvedi UC, Agarwal R, Elbishbishi EA et al. Cytokine cascade in dengue hemorrhagic fever: Implications for pathogenesis. FEMS Immunol Med Microbiol 2000; 28:183-88.

13. Moshage $H$, Kok B, Huizenga JR et al. Nitrite and nitrate determinations in plasma: A critical evaluation. Clin Chem 1995; 41: 892-96.

14. Boyde TRC, Rahmatulla M. Optimisation of conditions for the colorimetric determination of citruline using diacetylemonoxime. Anal Biochem 1980; 107: 424-31.

15. Torre D, Ferrario G, Speranza F et al. Increased levels of nitrite in the sera of children infected with human immunodeficiency virus type-1. Clin Infect Dis 1996; 22: 650-53.

16. Venugopalan A, Ghorpade RP, Chopra A. Cytokines in acute chikungunya. PLOS ONE 2014; 9: e111305. 
17. Barve S, Nanda S, Javadekar TB. Chikungunya fever: The resurgence and epidemiological pattern in western India. Nat J Med Res 2013; 3: 159-61.

18. Chikungunya: WHO fact sheet Apr 2016.

19. van de Weg CAM, Huits RMHG, Pannuti CS et al. Hyperferritinaemia in dengue virus infected patients is associated with immune activation and coagulation disturbances. PLoS Negl Trop Dis 2014; 8: e3214.

20. Pialoux G, Gauze 're BA, Jaure' guiberry $S$ et al. Chikungunya, an epidemic arbovirosis. Lancet 2007; 7: 319-27.

21. Uehara E.U., Shida BS, de Brito CA. Role of nitric oxide in immune responses against viruses: Beyond microbicidal activity. Inflamm Res 2015; 64: 845-52.

22. Dinarello CA. Historical review of cytokines. Eur J Immunol 2007; 37(suppl1): S34-S45.

23. Kim SY, Solomon DH. Tumor necrosis factor blockade and the risk of viral infection. Nat Rev Rheumatol 2010; 6: 165-74.

24. Chirathaworn C, Poovorawan Y, Lertmaharit S et al. Cytokine levels in patients with Chikungunya virus infection. Asia Pacific J Trop Med 2013; 6: 631-34.

25. Schroder K, Hertjog PJ, Ravasi $P$ et al. Interferon- $\psi$ :
An overview of signals, mechanisms and functions. J Leukocyte Biol 2004; 75: 163-89.

26. Mosser DM, Zhang X. Interleukin-10: New perspectives on an old cytokine. Immunol Rev 2008; 226: 205-18.

27. Thangamani S, Higgs S, Ziegler S et al. Host immune response to mosquito-transmitted Chikungunya virus differs from that elicited by needle inoculated virus. PLOS ONE 2010; 5: e12137.

28. Ng LFP, Chow A, Sun Y-J et al. IL-1b, IL-6, and RANTES as biomarkers of Chikungunya severity. PLoS One 2009; 4: e426.

29. Lum FM, Teo TH, Lee WWL et al. An essential role of antibodies in the control of Chikungunya virus infection. J Immunol 2013; 190: 6295-6302.

30. Kam Y, Lee WL, Simarmata D et al. Longitudinal analysis of the human antibody response to Chikungunya virus infection: implications for serodiagnosis and vaccine development. J Virol 2012; 86: 13005-15.

Date of Submission: 2017-07-24

Date of Acceptance: 2017-09-07 